\title{
Nobel Lecture: Type-II superconductors and the vortex lattice*
}

\author{
A. A. Abrikosov \\ Materials Science Division, Argonne National Laboratory, Argonne, Illinois 60439, USA
}

(Published 2 December 2004)

In 1950 Vitaly Ginzburg and Lev Landau published their famous paper on the theory of superconductivity (Ginzburg and Landau, 1950). The approach was based on the general theory of the second-order phase transitions proposed by Landau in 1937 (Landau, 1937). There Landau introduced the main variable, the so-called order parameter, which was finite below the transition and zero above it. Different phase transitions had different order parameters, and whereas it was evident for, e.g., the ferromagnetic transitions, namely, the spontaneous magnetization, it was far less evident for the superconducting transitions. Ginzburg and Landu had a stroke of genius when they chose, as the order parameter, some sort of wave function. At that time nobody knew about Cooper pairs or about their Bose condensates, where all particles become coherent, i.e., described by the same wave function. This assumption was the basis of the new theory, which managed to solve the main contradiction of the old theory by Fritz and Heinz London (1935), namely, the positive surface energy. Besides, it made many useful predictions, such as the critical magnetic field of thin films, the critical current in thin wires, etc.

All these predictions required experimental verification, and my friend and University mate, Nikolay Zavaritskii, started to measure the critical field of thin films. Theory and experiment fitted perfectly, including the change of the nature of the transition: first order at larger thicknesses and second order at smaller ones. Everything seemed OK but Alexander Shalnikov, Zavaritskii's boss, was not satisfied. He said that the films used by Zavaritskii were bad, since they were prepared at room temperature. The atoms of the metal, evaporated on a glass substrate, could agglomerate, and therefore the film actually consisted of small droplets. In order to avoid that, Shalnikov recommended maintaining the glass substrate at helium temperatures during evaporation and until the measurements were finished. Then every metal atom hitting the surface would stick to its place, and the film would be homogeneous.

Zavaritskii followed this advice, and the result was a surprise: the dependence of the critical field on the thickness, or temperature (the theory contains the ratio of the thickness to the penetration depth, which depends on temperature), did not fit the predictions of the

\footnotetext{
*The 2003 Nobel Prize in Physics was shared by A. A. Abrikosov, Vitaly L. Ginzburg, and Anthony J. Leggett. This lecture is the text of Dr. Abrikosov's address on the occasion of the award.
}

Ginzburg-Landau theory. Discussing these results with Zavaritskii, we could not believe that the theory was wrong: it was so beautiful and fitted so well the previous data. Therefore, we tried to find some solution in the framework of the theory itself, and we found it. The equations of the theory, where all entering quantities were expressed in corresponding units, depended only on one dimensionless "material" constant $\kappa$, which was later called the Ginzburg-Landau parameter. The value of $\kappa$ could be defined from the surface energy between the normal and superconducting phases. The latter, in its turn, could be calculated from the period of the structure of the intermediate state. These data for conventional superconductors led to very small values of $\kappa$, and therefore the calculations in the paper by Ginzburg and Landau were done for this limiting case. It was also established that with increasing value of $\kappa$ the surface energy between the superconducting and normal layers would become negative, and since this contradicted the existence of the intermediate state, such a case was not considered.

Therefore I decided to look at what would happen if $\kappa>1 / \sqrt{2}$, when the surface energy became negative. The transition in this case became second order for any thickness. The theory fitted completely Zavaritskii's experimental data, and this led us to the conclusion that there exists a special kind of superconductor, which we called "superconductors of the second group," with $\kappa>1 / \sqrt{2}$ and negative surface energy. Now they are called type-II superconductors. I published my derivation in the Russian journal Doklady Akademii Nauk SSSR in 1952 (Abrikosov, 1952). This was the earliest introduction of type-II superconductors. Since, however, this journal was never translated into English, there is a considerable confusion on this point, and the most common is just a statement that "there exist two types of superconductors... ." In Russia the idea of type-II superconductors raised no objections, although such materials were considered as exotic. In this connection it is worthwhile to mention that virtually all new superconducting compounds, discovered since the early 1960s up to the present time, are type-II superconductors. They include organics, A-15, Chevrel phaes, heavy fermionic materials, fullerenes, and high-temperature superconductors. One could say that now type-I superconductors have become exotic.

After the work on films I decided to look at the magnetic properties of bulk type-II superconductors. It was definite that the transition to the normal state in a mag- 
netic field would be of the second order, and the transition point defined by a stationary infinitesimal nucleus. Such nucleation fields were actually defined in the paper of Ginzburg and Landau. Their highest value corresponded in type-II superconductors to the so-called upper critical field $H_{c 2}$ :

$$
H_{c 2}=H_{c m} \kappa \sqrt{2},
$$

where $H_{c m}$ is the scale of magnetic fields, and it is defined as the critical field of a first-order transition of a type-I $(\kappa<1 / \sqrt{2})$ bulk cylinder in a longitudinal field.

At smaller magnetic fields one could imagine a linear combination of such nuclei centered at different points. Due to the homogeneity of space, the solution has to be periodic. Taking into account the necessity to renormalize the vector potential, one arrived at the following general expression for the order parameter:

$$
\Psi=\sum_{n=-\infty}^{\infty} C_{n} \exp \left[i k n y-\frac{1}{2} \kappa^{2}\left(x-\frac{k n}{\kappa^{2}}\right)^{2}\right] .
$$

Here and further the coordinates are measured in units of the penetration depth $\lambda$, and $k$ in $1 / \lambda$. The free energy becomes

$$
\frac{\Omega_{s}-\Omega_{n}^{(0)}}{H_{c m}^{2} / 4 \pi}=B^{2}-\frac{\kappa-B}{1+\left(2 \kappa^{2}-1\right) \beta_{A}},
$$

where $\Omega_{n}^{(0)}$ is the free energy of a normal metal in zero field, $B$ is the magnetic induction (average field), measured in units $H_{c m} \sqrt{2}$, and

$$
\beta_{A}=\frac{\overline{|\Psi|^{4}}}{\left(|\Psi|^{2}\right)^{2}} .
$$

This dimensionless constant depends only on the geometry of the array, i.e., on the relative values of the coefficients $C_{n}$ of Landau (1937).

According to Eq. (3), the choice must be such that $\beta_{A}$ is minimal. It can be shown that this minimal value is $\beta_{A}=1.16$, and it corresponds to the following selection: $C_{n+4}=C_{n}, C_{0}=C_{1}=-C_{2}=-C_{3}$, and $k=\kappa(\pi \sqrt{3})^{1 / 2}$. This function corresponds to a triangular lattice. A slightly larger value, $\beta_{A}=1.18$, characterizes the square lattice with equal coefficients $C_{n}=C$ and $k=\kappa(2 \pi)^{1 / 2}$. In the latter case it is easier to illustrate the properties of the solution. It can be represented as a theta function, namely,

$$
\Psi=C \exp \left(-\frac{1}{2} \kappa^{2} x^{2}\right) \vartheta_{3}\left[1 ;(2 \pi)^{1 / 2} \kappa i(x+i y)\right] .
$$

Using properties of the theta function it can be shown that under rotation of the coordinate system by $\pi / 2$ the function $\Psi$ is only multiplied by a phase factor $\exp \left(\mathrm{i} \kappa^{2} x y\right)$. Thus $|\Psi|^{2}$ has the symmetry of a square lattice.

At points $x=(\sqrt{2 \pi} / \kappa)(m+1 / 2), y=(\sqrt{2 \pi} / \kappa)(n+1 / 2)$, where $m$ and $n$ are integers, the function $\Psi$ vanishes. Near these points in polar coordinates

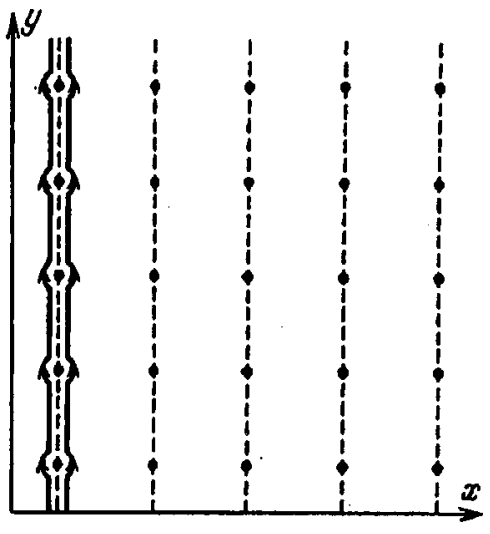

FIG. 1. The dots correspond to zeros of the order parameter (square lattice). The dashed lines are the branch cuts introduced in order to make the phase single valued. The gradient of the phase has a discontinuity at every branch cut (see text).

$$
\Psi \equiv|\Psi| e^{i \chi} \propto x+i y=\rho e^{i \varphi} .
$$

The phase $\chi=\varphi$, and hence it changes by $2 \pi$ along a contour around the zero of $\Psi$. A similar situation takes place in the case of a triangular lattice. The question naturally arises, how did it happen that the solution has these points? We just took a linear combination of the simple solutions, centered at different points, and the appearance of the zeros with phases changing by $2 \pi$ happened "by itself." In order to explain their appearance we have to take into account that in the GinzburgLandau equations the magnetic field is represented by the vector potential. If, on average, the magnetic field is constant, the vector potential has to grow with the coordinates. Since, however, the absolute value of the order parameter cannot have a systematic growth, the growth of the vector potential has to be compensated. This can be done by the phase of the order parameter.

If the phase is taken into account, i.e., $\Psi=|\Psi| e^{i \chi}$, then $\chi$ enters Ginzburg-Landau equations in the following combination with the vector potential:

$$
\mathbf{A}-\frac{\hbar c}{2 e} \nabla \chi
$$

Consider the behavior of the complex order parameter in the coordinate plane (Fig. 1). In order to define the phase unambiguously, branch cuts are introduced in this plane, going through the zeros of the order parameter parallel to the $y$ axis.

If we move along the left edge of such a branch cut, the phase varies according to the formula

$$
\chi_{l e f t}(y)=\chi_{r e g}-\pi \frac{y}{a},
$$

where the first term is regular part, and the second is due to the rapid change of the phase in the vicinity of a zero of $\Psi ; a$ is period. Along the right edge it changes according to the formula 


$$
\chi_{\text {right }}(y)=\chi_{r e g}+\pi \frac{y}{a}
$$

From these two expressions it can be concluded that the gradient of the phase has a discontinuity at every branch cut:

$$
\Delta\left(\frac{\partial \chi}{\partial y}\right)=\frac{2 \pi}{a} .
$$

If the magnetic field is directed along $z$, and we chose $A_{y}=H x$, then the compensation of the growth of the vector potential, according to Eq. (6), can be achieved if $H a=\pi \hbar c / e a$, or

$$
a=\sqrt{\frac{\pi \hbar c}{e H}} .
$$

If follows that

$$
H a^{2}=\frac{\pi \hbar c}{e} \equiv \Phi_{0}
$$

From these formulas two conclusions can be made: (a) The period of the structure grows with decreasing magnetic field, and (b) the flux of the magnetic field through one elementary cell is a universal constant, which is called the magnetic flux quantum. It is equal to 2.05 $\times 10^{-7}{\mathrm{Oe} \mathrm{cm}^{2}}^{2}$ and was first introduced by $\mathrm{F}$. London (1950).

The increase of the period with decreasing magnetic field happens not only close to $H_{c 2}$ but also at any field. Indeed, the reasoning leading to Fig. 1 and corresponding conclusions remains justified, except that the vector potential is no longer a linear function of the coordinate, and the compensation condition has to be reformulated. This leads to the replacement of the magnetic field by its average value $B=\left(1 / a^{2}\right) \int_{0}^{a} \int_{0}^{a} H d x d y$. Hence we get the same result as before, with $B$ instead of $H$.

From this it can be concluded that even far from $H_{c 2}$ the period of the structure increases with decreasing magnetic field, and its value, $H_{c 1}$, at which $B=0$ or $a$ $=\infty$, is the boundary between a purely superconducting phase and a phase with a partial penetration of the magnetic field, which I called a mixed state. The boundary with the purely superconducting phase corresponds to the magnetic field

$$
H_{c 1}=\frac{H_{c m}}{\kappa \sqrt{2}}(\ln \kappa+0.08) \text {. }
$$

According to Eq. (1), with increasing $\kappa$ the upper critical field $H_{c 2}$ grows, and simultaneously the lower critical field $H_{c 1}$ decreases.

Since the distance between the zeros of $\Psi$ becomes infinite at $H_{c 1}$, in its vicinity it is large, and only one such point can be considered. According to the GinzburgLandau theory, the current can be written in the form

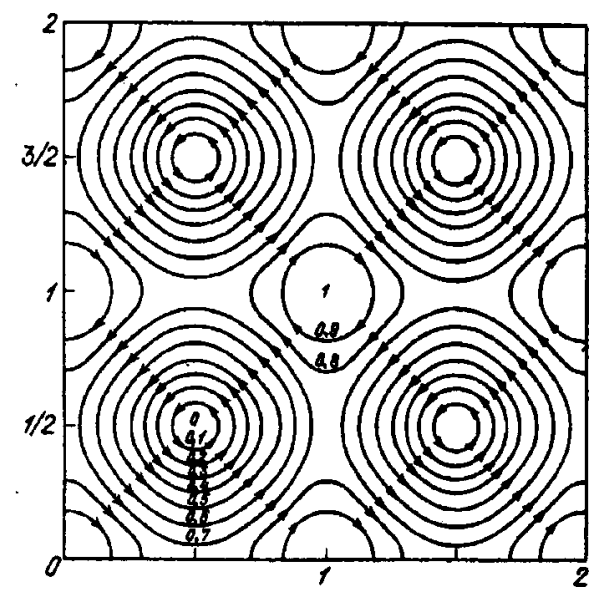

FIG. 2. The lines of current coinciding with the lines of constant $|\Psi|$ for a square lattice.

$$
\mathbf{j}=\frac{\hbar e}{\mathrm{~m}}|\Psi|^{2}\left(\nabla \chi-\frac{2 e}{\hbar c} \mathbf{A}\right)
$$

In the vicinity of $\Psi=0, \chi=\varphi$, and $\nabla \chi$ has only the $\varphi$ component, which is equal to $(1 / \rho)(\partial \chi / \partial \varphi)=1 / \rho$. Hence it is much larger than the second term in Eq. (12), and the current forms a vortex. In the general case these vortices form a lattice. The lines of the current in the vicinity of $H_{c 2}$ are presented in Fig. 2.

A very similar structure is the triangular lattice, which for an isotropic model has a slightly lower energy. Since the energy difference is very small, in real substances the crystalline symmetry can make the square lattice more favorable. Due to this structure, the mixed state is sometimes called the vortex lattice phase.

In the microscopic Bardeen-Cooper-Schrieffer (BCS) theory, as well as in the Ginzburg-Landau theory, which, as shown by Gor'kov $(1959,1960)$, is the limiting case of the BCS theory at $T \rightarrow T_{c}$, there exist two characteristic lengths: the smaller coherence length $\xi$, which is the size of a Cooper pair, and the larger penetration depth $\lambda$. The Ginzburg-Landau parameter $\kappa$ is, essentially, the ratio between these lengths. For a pure superconductor at $T$ $\rightarrow T_{c}$,

$$
\kappa=0.96 \frac{\lambda_{L}}{\xi_{0}},
$$

where $\lambda_{L}=\left(m c^{2} / 4 \pi n e^{2}\right)^{1 / 2}$ is the London penetration depth ( $n$ is the electron density), and $\xi_{0}=0.18\left(\hbar v / T_{c}\right)$ is the coherence length at $T=0$ ( $v$ is the electron velocity). In the case $\kappa \gg 1, \lambda \gg \xi$ (extreme type-II or London-type superconductor) every vortex has a "core" of size $\xi$, where the order parameter varies rapidly, and an outer region of size $\lambda$, where the magnetic field decays to zero. According to Eq. (6), in the vicinity of the vortex axis the order parameter grows linearly with distance. The vanishing of $\Psi$ at the center is due to the fact that this is the only way to avoid ambiguity of $\Psi$. Beyond distances of the order of $\xi$ the order parameter approaches the equilibrium value at zero field. The overall shape of the 


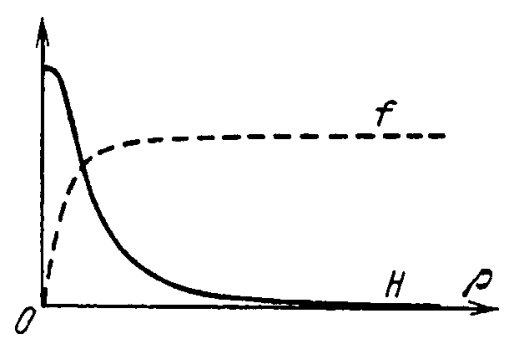

FIG. 3. Plot of the magnetic field (solid line) and $|\Psi|$ in a vortex.

behavior of the order parameter and magnetic field in a vortex is presented in Fig. 3.

The theory also allows us to define the macroscopic characteristics, namely, the dependence of the magnetization on external field. The latter is presented in Fig. 4 for different values of $\kappa$.

For $\kappa<1 / \sqrt{2}$ the dependence is a "triangle," reflecting an ideal diamagnetism below $H_{c m}$ and no magnetization in the normal phase. At higher values of $\kappa$ the vortex phase appears, and with increasing $\kappa$ its lower boundary decreases, whereas its upper boundary increases. The limiting formula for the magnetization in the vicinity of the upper critical field is

$$
-4 \pi M=\frac{H_{c 2}-H_{0}}{\left(2 \kappa^{2}-1\right) \beta_{A}} .
$$

I compared the theoretical predictions about the magnetization curves with the experimental results obtained by Lev Shubnikov and his associates on $\mathrm{Pb}-\mathrm{Tl}$ alloys in 1938 (Shubnikov et al., 1937), and there was a very good fit.

Here I would like to describe the situation with experiments. The magnetization of superconducting alloys was first measured by de Haas and Casimir-Jonker in 1935 (Casimir-Jonker and de Haas, 1935), and they got a gradual transition from the superconducting to the normal state with two critical fields. They explained this by the inhomogeneity of their samples. Shubnikov, who worked previously with de Haas, decided to make better samples, and his group annealed the alloys a long time at temperatures close to the melting point. After that the $\mathrm{x}$-ray diffraction studies, performed at room temperature, did not show any inhomogeneity. Since the authors could not imagine another explanation for the gradual transition, they wrote in their paper that the precipitation of another phase must happen at lower temperature. Unfortunately, L. V. Shubnikov was accused of attempting to organize an "anti-Soviet strike," arrested

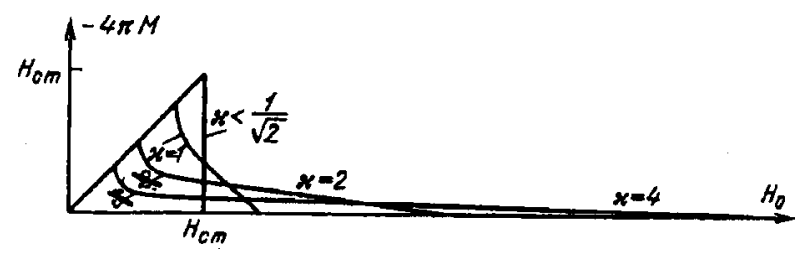

FIG. 4. Dependence of magnetization on magnetic field for different values of $\kappa$.

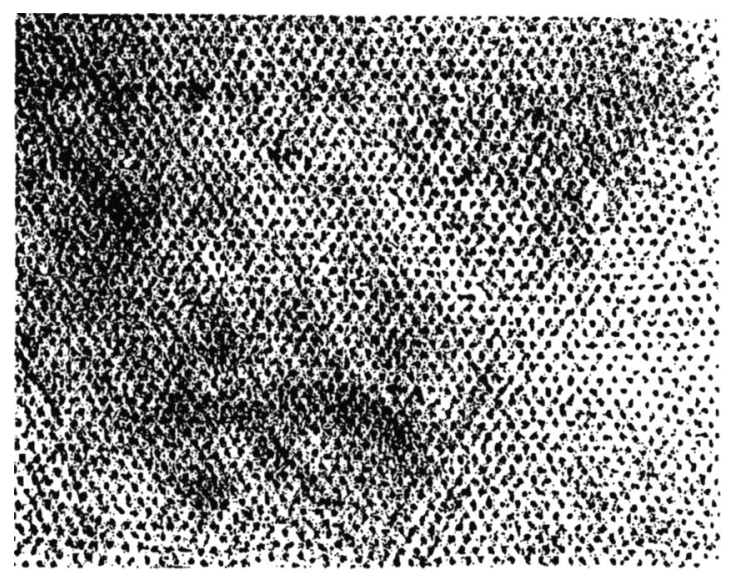

FIG. 5. First decoration picture of vortices by Essmann and Traeuble (1967).

and executed by the KGB the same year. I am sure that, given the opportunity, he would have discovered the appearance of a new phase and the existence of a special kind of superconductor. I would like here to pay a tribute to Shubnikov, whose data gave me real inspiration. I never met him but I heard about him from Landau, who was his close friend.

I made my derivation of the vortex lattice in 1953 but publication was postponed since Landau at first disagreed with the whole idea. Only after R. Feynman published his paper on vortices in superfluid helium (Feynman, 1955), and Landau accepted the idea of vortices, did he agree with my derivation, and I published my paper (Abrikosov, 1957). Even then it did not attract attention, in spite of an English translation. Only after the discovery in the beginning of the sixties of superconducting alloys and compounds with high critical magnetic fields did there appear an interest in my work, and even after that the experimentalists did not believe in the possibility of the existence of a vortex lattice incommensurable with the crystalline lattice. When the vortex lattice was observed experimentally, first by neutron diffraction (Cribier et al., 1964) and then by decoration (Essmann and Traeuble, 1967; see Fig. 5) they had no more doubts. Now there exist many different ways to get images of the vortex lattice. Apart from those already mentioned, there are electron holography, scanning tunneling microscopy (Fig. 6), and magneto-optics.

After that I made only one more study of vortices, namely, I defined the lower critical field of thin films and the vortex lattice in its vicinity (Abrikosov, 1964).

Although I worked in many different fields of theoretical physics afterwards, superconductivity was my favorite. In the beginning of the sixties we worked on several projects together with Lev Gor'kov. These were based on his Green's-function presentation of the BCS theory, which permitted one to extend the microscopic theory to spatially inhomogeneous problems. We studied the behavior of superconductors in a high-frequency field (Abrikosov, Gor'kov, and Khalatnikov, 1958, 1959), the role of magnetic impurities (Abrikosov and Gor'kov, 1961), where we discovered the so-called "gapless" su- 


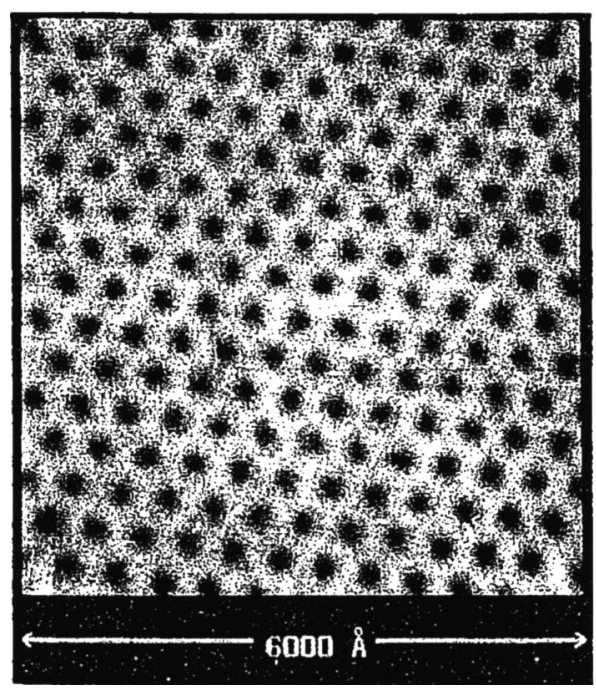

FIG. 6. Vortices in $\mathrm{NbSe}_{2}$ defined by scanning tunneling microscopy (STM).

perconductivity, and managed to solve the mystery of the finite Knight shift at low temperatures, introducing the spin-orbit scattering (Abrikosov and Gor'kov, 1962).

After the discovery of high-temperature superconductivity in layered copper oxides by J. G. Bednorz and K. A. Mueller (1986) I became interested in their properties. There existed many different approaches to these unusual substances and virtually all of them postulated some exotic mechanism of superconductivity. I based my approach on the BCS theory, taking into account the specific features of the electron spectrum, mostly the quasi-two-dimensionality and the so-called "extended saddle point singularities," or "flat regions" in the electron spectrum (Abrikosov, 2000). Another idea was the resonant tunneling connection between the $\mathrm{CuO}_{2}$ layers (Abrikosov, 1999), which is responsible for conductivity and superconductivity. On this basis I was able to ex- plain most of the experimental data about layered cuprates without dividing them into "good" ones, which should be mentioned on every possible occasion, and "bad" ones, which should be forgotten. As a result I can state that the so-called "mystery" of high- $T_{c}$ superconductivity does not exist.

\section{REFERENCES}

Abrikosov, A. A., 1952, Dokl. Akad. Nauk SSSR 86, 489.

Abrikosov, A. A., 1957, Sov. Phys. JETP 5, 1174.

Abrikosov, A. A., 1964, Sov. Phys. JETP 19, 988.

Abrikosov, A. A., 1999, Physica C 317-318, 154.

Abrikosov, A. A., 2000, Physica C 341-348, 97.

Abrikosov, A. A., and L. P. Gor'kov, 1961, Sov. Phys. JETP 12, 1243.

Abrikosov, A. A., and L. P. Gor'kov, 1962, Sov. Phys. JETP 15, 752.

Abrikosov, A. A., L. P. Gor'kov, and I. M. Khalatnikov, 1958, Sov. Phys. JETP 8, 182; 10, 132 (1959).

Bednorz, J. G., and K. A. Mueller, 1986, Z. Phys. B: Condens. Matter 64, 189.

Casimir-Jonker, J. M., and W. J. de Haas, 1935, Physica (Amsterdam) 2, 943.

Cribier, D., B. Jacrot, L. M. Rao, and B. Farnoux, 1964, Phys. Lett. 9, 106.

Essmann, U., and H. Traeuble, 1967, Phys. Lett. 24A, 526.

Feynman, R. P., 1955, in Progress in Low Temperature Physics, edited by D. F. Brewer (North-Holland, Amsterdam), Vol. 1, Chap. 11.

Ginzburg, V. L., and L. D. Landau, 1950, Zh. Eksp. Teor. Fiz. 20, 1064.

Gor'kov, L. P., 1959, Sov. Phys. JETP 9, 1364; 10, 998 (1960).

Landau, L. D., 1937, Phys. Z. Sowjetunion 11, 26; 11, 129 (1937).

London, F., 1950, Superfluids (New York), Vol. 1.

London, F., and H. London, 1935, Proc. R. Soc. London, Ser. A 149, 71.

Sbubnikov, L. V., et al., 1937, Zh. Eksp. Teor. Fiz. 7, 221. 